\title{
ARTICLE
}

Clinical Study

\section{A multicentre, randomised, open-label, parallel-group Phase $2 b$ study of belotecan versus topotecan for recurrent ovarian}

\section{cancer}

\author{
Hee Seung Kim ${ }^{1}$, Sang-Yoon Park ${ }^{2}$, Chan-Yong Park ${ }^{3}$, Young Tae Kim ${ }^{4}$, Beob-Jong Kim ${ }^{5}$, Yong Jung Song ${ }^{6}$, Byoung-Gie Kim7, \\ Yong Beom Kim ${ }^{8}$, Chi-Heum Cho ${ }^{9}$, Jong-Hyeok Kim ${ }^{10}$ and Yong Sang Song ${ }^{1}$
}

BACKGROUND: This Phase $2 \mathrm{~b}$ study compared the efficacy and toxicity of belotecan and topotecan in recurrent ovarian cancer. METHODS: Patients with platinum-sensitive recurrent or platinum-resistant recurrent ovarian cancer (PRROC) were randomised 1:1 to receive belotecan $0.5 \mathrm{mg} / \mathrm{m}^{2}$ or topotecan $1.5 \mathrm{mg} / \mathrm{m}^{2}$ for five consecutive days every 3 weeks. The primary endpoint was overall response rate (ORR); secondary endpoints were progression-free survival (PFS), overall survival (OS), and toxicity.

RESULTS: A total of 140 (belotecan, $n=71$; topotecan, $n=69$ ) and 130 patients (belotecan, $n=66$; topotecan, $n=64$ ) were included in the intention-to-treat (ITT) and per-protocol (PP) populations. ORR did not differ significantly between the belotecan and topotecan groups (ITT, 29.6\% versus 26.1\%; PP, 30.3\% versus 25\%). Although PFS did not differ between the groups, belotecan was associated with improved OS compared with topotecan in the PP population (39.7 versus 26.6 months; $P=0.034$ ). In particular, belotecan showed longer OS in PRROC and non-high-grade serous carcinoma (non-HGSC; PP, adjusted hazard ratios, 0.499 and $0.187 ; 95 \%$ confidence intervals $0.255-0.977$ and $0.039-0.895$ ). Furthermore, there were no differences in toxicities between the two groups.

CONCLUSIONS: Belotecan was not inferior to topotecan in terms of overall response for recurrent ovarian cancer.

CLINICAL TRIAL REGISTRATION: NCT01630018.

British Journal of Cancer (2021) 124:375-382; https://doi.org/10.1038/s41416-020-01098-8

\section{INTRODUCTION}

Ovarian cancer has a poor prognosis, with a 5-year survival rate of $<30 \%$ in stage IIIC-IV disease, with which more than two-thirds of patients are diagnosed. ${ }^{1}$ Despite a rate of complete response (CR) of $70-80 \%$ after primary treatment of advanced ovarian cancer, about $70 \%$ of patients ultimately relapse, and most of them die due to progression. ${ }^{2}$

Among patients showing disease recurrence, those with who relapse $>6$ months after the completion of primary chemotherapy are classified as platinum-sensitive recurrent ovarian cancer (PSROC), and platinum-based chemotherapy remains effective for them, with a response rate of up to $65 \% .^{3}$ In contrast, those with platinum-resistant recurrent ovarian cancer (PRROC), defined as recurrence $\leq 6$ months after treatment, show a lower response rate of $<30 \%$, despite the use of non-platinum agents. ${ }^{4}$

Although targeted agents including bevacizumab and poly ADP ribose polymerase (PARP) inhibitors and immune-oncologic agents including programmed death receptor-1 (PD-1) and programmed death-ligand 1 (PD-L1) inhibitors have been introduced to overcome drug resistance, some of them are still combined with conventional cytotoxic drugs for treating recurrent ovarian cancer in clinical trials.

Belotecan is a semi-synthetic camptothecin analogue with the water-solubilising group at position 7 unlike topotecan, which inhibits the relegation of single-stranded DNA breaks by blocking topoisomerase I, and thereby disrupting DNA replication and inducing apoptosis of tumour cells., ${ }^{5,6}$ In preclinical studies, camptothecin including belotecan showed the inhibition of type 1 DNA topoisomerase in S phase cells, which caused the arrest of replication forks and led to cell killing.,8 Moreover, these characteristics have been suggested to contribute to over twofold anti-tumour index of belotecan in comparison to topotecan, ${ }^{5}$ which can be related with better tumour response in ovarian cancer. ${ }^{9}$

\footnotetext{
${ }^{1}$ Department of Obstetrics and Gynecology, Seoul National University College of Medicine, Seoul 03080, Republic of Korea; ${ }^{2}$ Center for Uterine Cancer, Research Institute and Hospital, National Cancer Center, Goyang 10408, Republic of Korea; ${ }^{3}$ Department of Obstetrics and Gynecology, Gil Medical Center, Gachon University of Medicine and Science, Incheon 21565, Republic of Korea; ${ }^{4}$ Department of Obstetrics and Gynecology, Institute of Women's Medical Life Science, Yonsei University College of Medicine, Seoul 03722, Republic of Korea; ${ }^{5}$ Department of Obstetrics and Gynecology, Korea Cancer Center Hospital, Korea Institute of Radiological and Medical Sciences, Seoul 01812, Republic of Korea; ${ }^{6}$ Department of Obstetrics and Gynecology, Pusan National University School of Medicine, Yangsan 50612, Republic of Korea; ${ }^{7}$ Department of Obstetrics and Gynecology, Samsung Medical Center, Sungkyunkwan University School of Medicine, Seoul 06351, Republic of Korea; ${ }^{8}$ Department of Obstetrics and Gynecology, Seoul National University Bundang Hospital, Seongnam 13620, Republic of Korea; ${ }^{9}$ Department of Obstetrics and Gynecology, Dongsan Medical Center, Keimyung University, Daegu 42601, Republic of Korea and ${ }^{10}$ Department of Obstetrics and Gynecology, University of Ulsan College of Medicine, Asan Medical Center, Seoul 05505, Republic of Korea Correspondence: Yong Sang Song (yssong@snu.ac.kr)
} 
In clinical studies, belotecan-based chemotherapy has also been reported to show tumour response rates of $53-78 \%$ in PSROC and $20-50 \%$ in PRROC, ${ }^{10,11}$ which are higher than the tumour responses seen with topotecan or topotecan plus cisplatin of $17-33 \%$ in PSROC, ${ }^{12,13}$ and $14-19 \%$ in PRROC. ${ }^{14,15}$ These findings suggest the possibility that belotecan may have greater potential than topotecan to improve prognosis in recurrent ovarian cancer. Thus, we performed a Phase $2 b$ study to evaluate whether belotecan might be more effective than topotecan, with acceptable toxicity, in recurrent ovarian cancer, and thereby to assess the potential value of using it as a combination drug that could increase anti-cancer activity in the era of targeted and immuno-oncologic therapy.

\section{MATERIALS AND METHODS}

Study design

This was a multicentre, open-label, randomised, Phase $2 b$, noninferiority study comparing the efficacy and toxicity of belotecan with that of topotecan in recurrent ovarian cancer (NCT01630018). The protocol was approved by Institutional Review Boards at Seoul National University Hospital, National Cancer Center, Gil Medical Center, Severance Hospital, Korea Cancer Center Hospital, Pusan National University Yangsan Hospital, Samsung Medical Center, Seoul National University Bundang Hospital, Dongsan Medical Center and Asan Medical Center before the start of the study. The manuscript was written in accordance with the CONSORT 2010 reporting guideline.

Eligibility criteria were as follows: $\geq 18$ years of age; epithelial ovarian cancer; recurrent disease; measurable or non-measurable disease based on RECIST version $1.1^{16}$ or GCIG criteria; ${ }^{17}$ ECOG performance status $\leq 2$; remaining life expectancy of $>3$ months; normal hepatic, renal and bone marrow function. We excluded patients with: active bacterial infection requiring intravenous antibiotics; brain metastasis; synchronous or metachronous malignancies other than epithelial ovarian cancer during the previous 5 years, except basal cell carcinoma of the skin or carcinoma in situ of the cervix that had been treated appropriately; and prior anticancer treatment within 4 weeks of enrolment.
Treatment

All patients were assigned randomly $1: 1$ to receive either belotecan or topotecan. Belotecan $0.5 \mathrm{mg} / \mathrm{m}^{2}$ or topotecan 1.5 $\mathrm{mg} / \mathrm{m}^{2}$ was administered as a 30-min infusion intravenously for five consecutive days every 3 weeks for six cycles or until disease progression, if earlier. Moreover, additional chemotherapy was administered according to the discretion of investigators if clinical benefit such as $C R$ or partial response (PR) or stable disease was observed after the planed treatment.

When patients showed grade 3 or 4 haematologic or nonhaematologic toxicities, the cycle of chemotherapy was delayed by up to 2 weeks. If grade 3 or 4 toxicities persisted after 2 weeks, that cycle of chemotherapy was cancelled, and the next cycle was administered according to the planned schedule. Dose reduction was considered when patients showed febrile neutropenia, grade 2 neutropenia, or grade 1 thrombocytopenia with delayed chemotherapy within 2 weeks. Dose reduction to belotecan 0.1 $\mathrm{mg} / \mathrm{m}^{2} /$ day or topotecan $0.25 \mathrm{mg} / \mathrm{m}^{2} /$ day could be performed up to two times; administration was discontinued if an additional dose reduction was required.

\section{Endpoints}

The primary endpoint was overall response rate (ORR), the ratio of patients with $\mathrm{CR}$ or PR among all patients. Secondary endpoints were progression-free survival (PFS; from the time of randomisation to the time of confirmation of disease progression or death) and overall survival (OS; from the time of randomisation to the time of confirmation of death or the end of the study). Rates of adverse events were also compared, based on CTCAE version 4.0. ${ }^{18}$

Efficacy was compared between belotecan and topotecan using the intention-to-treat (ITT) population (those who were originally allocated after randomisation and underwent one or more evaluation of response) and the per-protocol (PP) population (patients who completed the treated originally allocated). Safety was compared between belotecan and topotecan using the ITT population.

\section{Statistical analysis}

For calculating the study sample size, ORR we selected as the primary endpoint because ORR was used as the primary endpoint

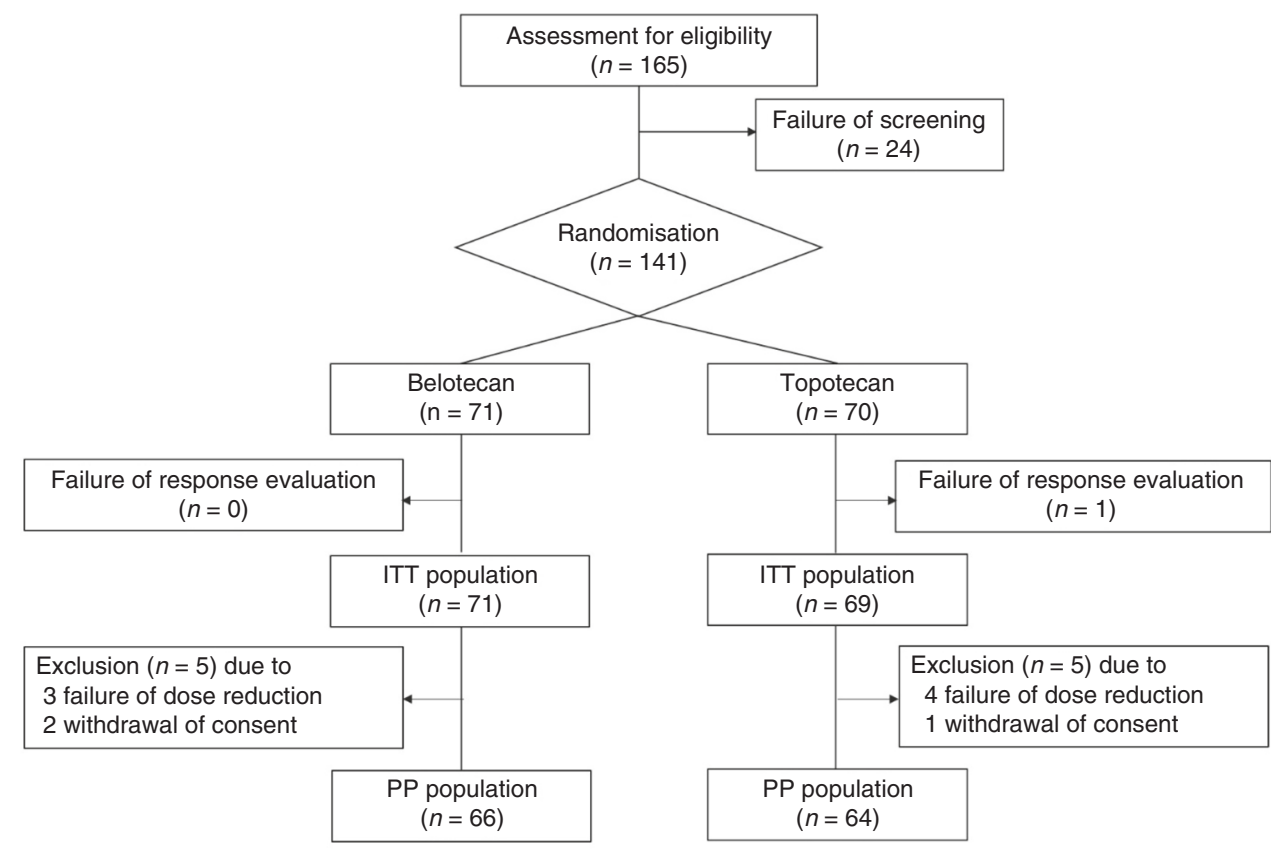

$\mathrm{ITT}=$ intention-to-treat, $\mathrm{PP}=$ per-protocol

Fig. 1 Patient disposition (CONSORT diagram). 
A multicentre, randomised, open-label, parallel-group Phase $2 b$ study of... HS Kim et al.

\begin{tabular}{|c|c|c|c|}
\hline Characteristics & $\begin{array}{l}\text { Belotecan } \\
(n=71, \%)\end{array}$ & $\begin{array}{l}\text { Topotecan } \\
(n=69, \%)\end{array}$ & $P$-value \\
\hline Age (mean, SD, years) & $54.5 \pm 10.6$ & $56.9 \pm 10.3$ & 0.099 \\
\hline FIGO stage & & & 0.294 \\
\hline I & $6(8.5)$ & $4(5.8)$ & \\
\hline II & $3(4.2)$ & $3(4.3)$ & \\
\hline III & $36(50.7)$ & $47(68.2)$ & \\
\hline IV & $26(36.6)$ & $15(21.7)$ & \\
\hline Histology & & & 0.324 \\
\hline High-grade serous & $49(69.1)$ & $57(82.7)$ & \\
\hline Endometrioid & $6(8.5)$ & $2(2.9)$ & \\
\hline Mucinous & $4(5.6)$ & $2(2.9)$ & \\
\hline Clear cell & $4(5.6)$ & $5(7.2)$ & \\
\hline Undifferentiated & $4(5.6)$ & $2(2.9)$ & \\
\hline Mixed & $4(5.6)^{a}$ & $1(1.4)^{\mathrm{b}}$ & \\
\hline $\begin{array}{l}\text { Number of prior } \\
\text { chemotherapy lines }\end{array}$ & & & 0.480 \\
\hline 1 & $37(52.1)$ & $34(49.3)$ & \\
\hline 2 & $25(35.2)$ & $29(42)$ & \\
\hline 3 & $7(9.9)$ & $6(8.7)$ & \\
\hline 4 & $2(2.8)$ & $0(0)$ & \\
\hline Additional chemotherapy & & & 0.275 \\
\hline No & $65(91.5)$ & $67(97.1)$ & \\
\hline Yes & $6(8.5)$ & $2(2.9)$ & \\
\hline Type of recurrence & & & 0.408 \\
\hline PSROC & $26(36.6)$ & $30(43.5)$ & \\
\hline PRROC & $45(63.4)$ & $39(56.5)$ & \\
\hline $\begin{array}{l}\text { Duration of follow-up } \\
\text { (mean, SD, months) }\end{array}$ & $18.7 \pm 11.1$ & $16.8 \pm 9.7$ & 0.297 \\
\hline \multicolumn{4}{|c|}{$\begin{array}{l}\text { FIGO International Federation of Gynecology and Obstetrics, PRROC } \\
\text { platinum-resistant recurrent ovarian cancer, PSROC platinum-sensitive } \\
\text { recurrent ovarian cancer, SD standard deviation. } \\
\text { aThree and one patient showed mixed high-grade serous/transitional cell } \\
\text { carcinoma and mixed high-grade serous/clear cell/transitional cell carci- } \\
\text { noma, respectively. } \\
\text { bone patient showed mixed high-grade serous/clear cell carcinoma. }\end{array}$} \\
\hline
\end{tabular}

in most previous trials with topotecan. ${ }^{12-14,19,20}$ This study was designed as a non-inferiority trial, and the ORR of topotecan reported for previous studies was considered when selecting the non-inferiority margin. The highest reported response rate for topotecan was $33 \%,{ }^{13}$ and the lowest response rate was about $14 \%$ with $95 \%$ confidence interval $(\mathrm{Cl})$ of $8-19 \%$. $^{19,20}$ Thus, the non-inferiority margin was determined as $25 \%$, calculated by subtracting the lowest margin of the $\mathrm{Cl}$ of the lowest response rate $(8 \%)$ from the highest response rate $(33 \%)$. A total of 140 subjects, comprising 70 per group, were required, assuming the power of $80 \%$, a type I error rate of $5 \%$ and a dropout rate of $20 \%$.

Dichotomous data were analysed using the $x^{2}$ or Fisher's exact test, and continuous variables were compared using the Student $t$ or Mann-Whitney $U$ test, comparing the experimental and control groups. Time-to-event variables were evaluated using Kaplan-Meier analysis with the log-rank or Breslow test. Independent prognostic factors were identified using Cox proportional hazard models with a hazard ratio (HR) and 95\% Cl. Statistical analyses were performed using SPSS software version 23.0 (Biostat Inc., Englewood, NJ, USA). $P<0.05$ was considered significant.

\begin{tabular}{|c|c|c|c|}
\hline Overall response rate & Belotecan & Topotecan & $P$-value \\
\hline \multicolumn{4}{|l|}{ ITT population $(n / N, \%)$} \\
\hline All patients & 21/71 (29.6) & $18 / 69(26.1)$ & 0.645 \\
\hline \multicolumn{4}{|l|}{ Type of recurrence } \\
\hline PSROC & $10 / 26(38.5)$ & 10/30 (33.3) & 0.783 \\
\hline PRROC & $11 / 45(24.4)$ & $8 / 39(20.5)$ & 0.795 \\
\hline \multicolumn{4}{|l|}{ Histology } \\
\hline HGSC & $14 / 49(28.6)$ & $17 / 40(29.8)$ & 1.000 \\
\hline Non-HGSC & $7 / 22(31.8)$ & $1 / 12(8.3)$ & 0.210 \\
\hline \multicolumn{4}{|l|}{ PP population $(n / N, \%)$} \\
\hline All patients & 20/66 (30.3) & $16 / 64(25)$ & 0.499 \\
\hline \multicolumn{4}{|l|}{ Type of recurrence } \\
\hline PSROC & $10 / 24(41.7)$ & 9/26 (34.6) & 0.608 \\
\hline PRROC & $10 / 42(23.8)$ & 7/38 (18.4) & 0.556 \\
\hline \multicolumn{4}{|l|}{ Histology } \\
\hline Serous & $15 / 53(28.3)$ & $13.45(28.9)$ & 1.000 \\
\hline Non-serous & $7 / 21(33.3)$ & $1 / 11(9.1)$ & 0.209 \\
\hline
\end{tabular}

HGSC high-grade serous carcinoma, ITT intention-to-treat, $P P$ per-protocol, $P R R O C$ platinum-resistant recurrent ovarian cancer, PSROC platinumsensitive recurrent ovarian cancer.

\section{RESULTS}

Population

A total of 141 patients were enrolled between January 2011 and June 2014, of whom 140 and 130 patients were included in ITT and PP populations after exclusion of 10 patients due to the protocol violation such as failure of dose reduction $(n=7)$ and withdrawal of consent $(n=3)$ (Fig. 1). Table 1 shows that clinicopathologic characteristics were similar between the belotecan and topotecan groups. Additional chemotherapy up to nine cycles was given in eight patients (5.7\%) with high-grade serous carcinoma (HGSC). Among them, two with PSROC (2.8\%) and four with PRROC (5.6\%) received it in the belotecan group, whereas one with PSROC (1.4\%) and one with PRROC (1.4\%) received in the topotecan group. The rates of cancellation and dose reduction and delay of chemotherapy at each cycle were not different between the two groups (Supplementary Table 1).

Efficacy

CR was not seen in the study, and ORR did not differ between the belotecan and topotecan groups in either the ITT $(29.6 \%$ versus $26.1 \%, P=0.645)$ or PP (30.3\% versus $25.0 \%, P=0.499)$ populations, which suggest that belotecan was not inferior to topotecan. In terms of the type of recurrence, ORR did not differ between the belotecan and topotecan groups among those with PSROC (ITT, $38.5 \%$ versus $33.3 \%$; PP, $41.7 \%$ versus $34.6 \% ; P>0.05$ ) or those with PRROC (ITT, $24.4 \%$ versus $20.5 \%$; PP, $23.8 \%$ versus $18.4 \% ; P>$ $0.05)$. Moreover, ORR also did not differ between the groups among those with high-grade serous carcinoma (HGSC; ITT, 28.6\% versus $29.8 \%$; PP, $28.3 \%$ versus $28.9 \%$; $P>0.05$ ) or non-HGSC (ITT, $31.8 \%$ versus $8.3 \%$; PP, $33.3 \%$ versus $9.1 \% ; P>0.05$; Table 2 ).

With respect to survival, there was no difference in PFS between the two groups (Supplementary Fig. 1). On the other hand, belotecan was associated with improved OS with marginal significance in the ITT population (median 31.5 versus 22.9 months, $P=0.073$ ), with the relation becoming stronger in the PP population (median 39.7 versus 26.6 months; $P=0.034$ ). There was no difference in OS between the two treatment groups among those with PSROC; however, belotecan was related to improved OS with marginal significance in the PP population among those with PRROC (median 31.5 versus 15.5 months, 

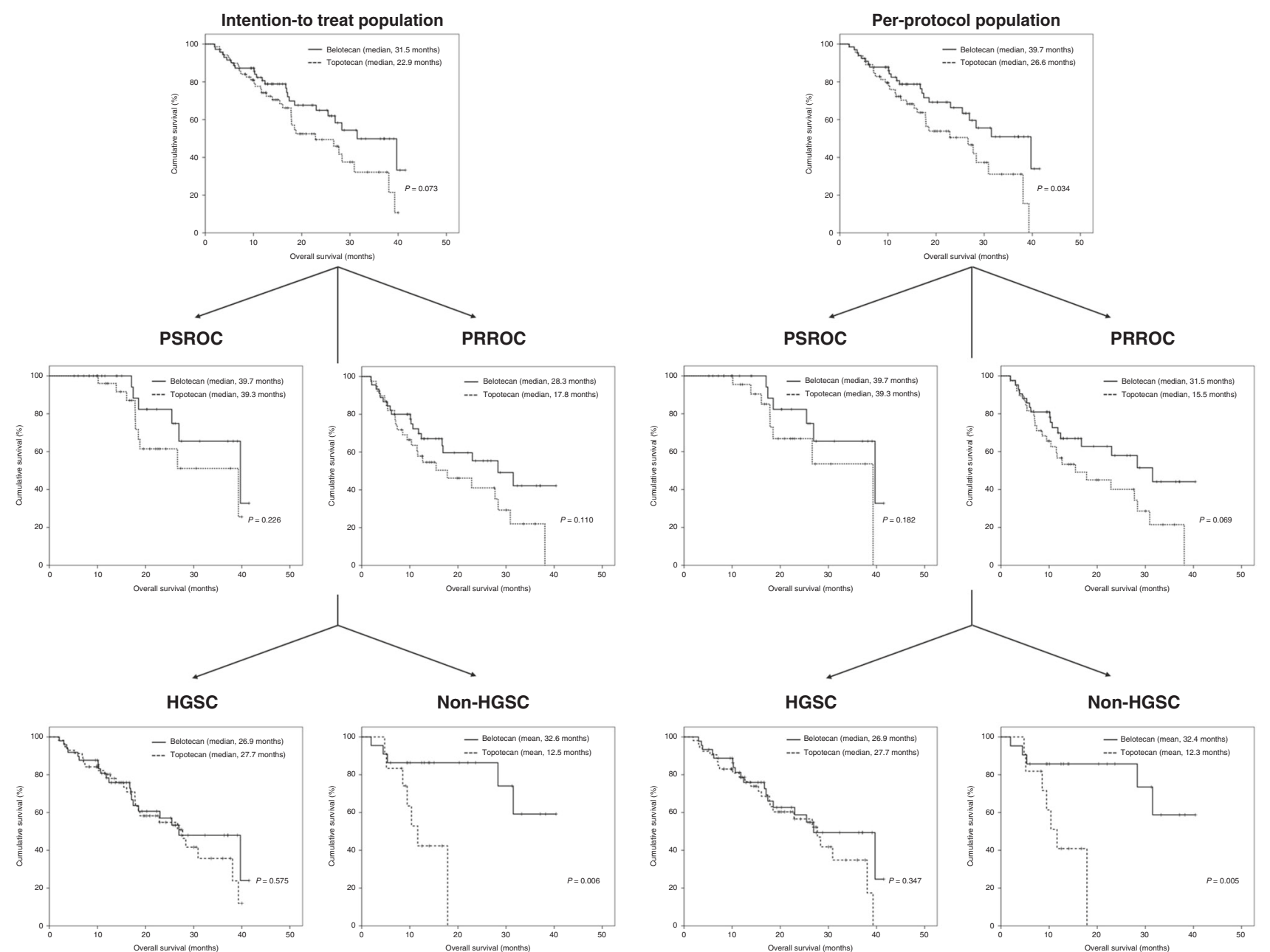

Fig. 2 Comparison of overall survival between belotecan and topotecan in intention-to-treat and per-protocol populations. Subgroup analyses were conducted according to the type of recurrence (platinum-sensitive recurrent ovarian cancer, PSROC; platinum-resistant recurrent ovarian cancer, PRROC) and histology (high-grade serous carcinoma, HGSC; non-HGSC).

$P=0.069)$. Moreover, belotecan was also associated with prolonged OS in those with non-HGSC (ITT, mean 32.6 versus 12.5 months; PP, 32.4 versus 12.3 months; $P<0.05$; Fig. 2 ).

In multivariate analyses, PSROC and additional chemotherapy were prognostic factors for PFS in patients with HGSC (ITT, adjusted HRs, 0.516 and $0.284 ; 95 \% \mathrm{Cls}, 0.325-0.818$ and $0.106-0.759 ;$ PP, 0.483 and $0.263 ; 0.297-0.785$ and $0.097-0.711$ ), and PSROC was the only prognostic factor for PFS in those with non-HGSC (ITT, adjusted HR, 0.285; 95\% Cl, 0.103-0.878; PP, 0.265; $0.086-0.813)$. However, belotecan was not a prognostic factor for PFS (Supplementary Tables 2 and 3).

On the other hand, PSROC was a prognostic factor for improving OS in those with HGSC (ITT, adjusted HR, 0.426; 95\% $\mathrm{Cl}, 0.221-0.820 ; \mathrm{PP}, 0.421 ; 0.209-0.847)$ and those with non-HGSC (PP, adjusted HR, 0.156; 95\% Cl, 0.019-0.953). Moreover, belotecan was a favourable prognostic factor for improved OS in patients with PRROC (PP, adjusted HR 0.499; 95\% Cl 0.255-0.977; $P=$ 0.043 ), and those with non-HGSC (ITT, adjusted $\mathrm{HR}, 0.185 ; 95 \% \mathrm{Cl}$, 0.039-0.890; PP, 0.187; 0.039-0.895; Table 3).

In particular, 10 patients with endometrioid $(n=6)$ or clear cell carcinoma $(n=4)$ who received belotecan were alive, whereas 4 of $7(57.1 \%)$ with endometrioid $(n=2)$ or clear cell carcinoma $(n=$ 5) who received topotecan died during this study. When we performed subgroup analyses for the patients with endometrioid or clear cell carcinoma, there were no differences in clinicopathologic characteristics between the belotecan and topotecan groups
(Supplementary Table 4). In terms of survival, belotecan was also associated with improved OS despite no difference in PFS between the two groups in either the ITT or PP populations (Supplementary Fig. 2).

Safety

With respect to haematologic toxicity, grade 3 or 4 neutropenia and anaemia were observed commonly in the belotecan and topotecan groups (grade 3: $64.8 \%$ versus $53.6 \%$, and $15.5 \%$ versus $20.3 \%$; grade $4: 52.1 \%$ vs. $39.1 \%$, and $1.4 \%$ versus $0 \%$ ). In terms of non-haematologic toxicity, grade 3 diarrhoea and ileus were relatively common in the belotecan and topotecan groups ( $4.2 \%$ versus $0.0 \%$, and $0.0 \%$ versus $2.9 \%$ ). However, there were no differences in both haematologic and non-haematologic toxicities between the belotecan and topotecan groups (Table 4).

\section{DISCUSSION}

Topoisomerase I inhibitors are popular anti-cancer drugs that interrupt the ligation step of the cancer cell cycle, generating single or double-stranded breaks that harm the integrity of the genome, leading to apoptosis or cell death. Although bevacizumab or PARP inhibitors combined with taxane- and platinumbased chemotherapy have been shown to be effective for treating PSROC $^{21,22}$ the cytotoxic effects of topoisomerase I inhibitors are 
A multicentre, randomised, open-label, parallel-group Phase $2 b$ study of... HS Kim et al.

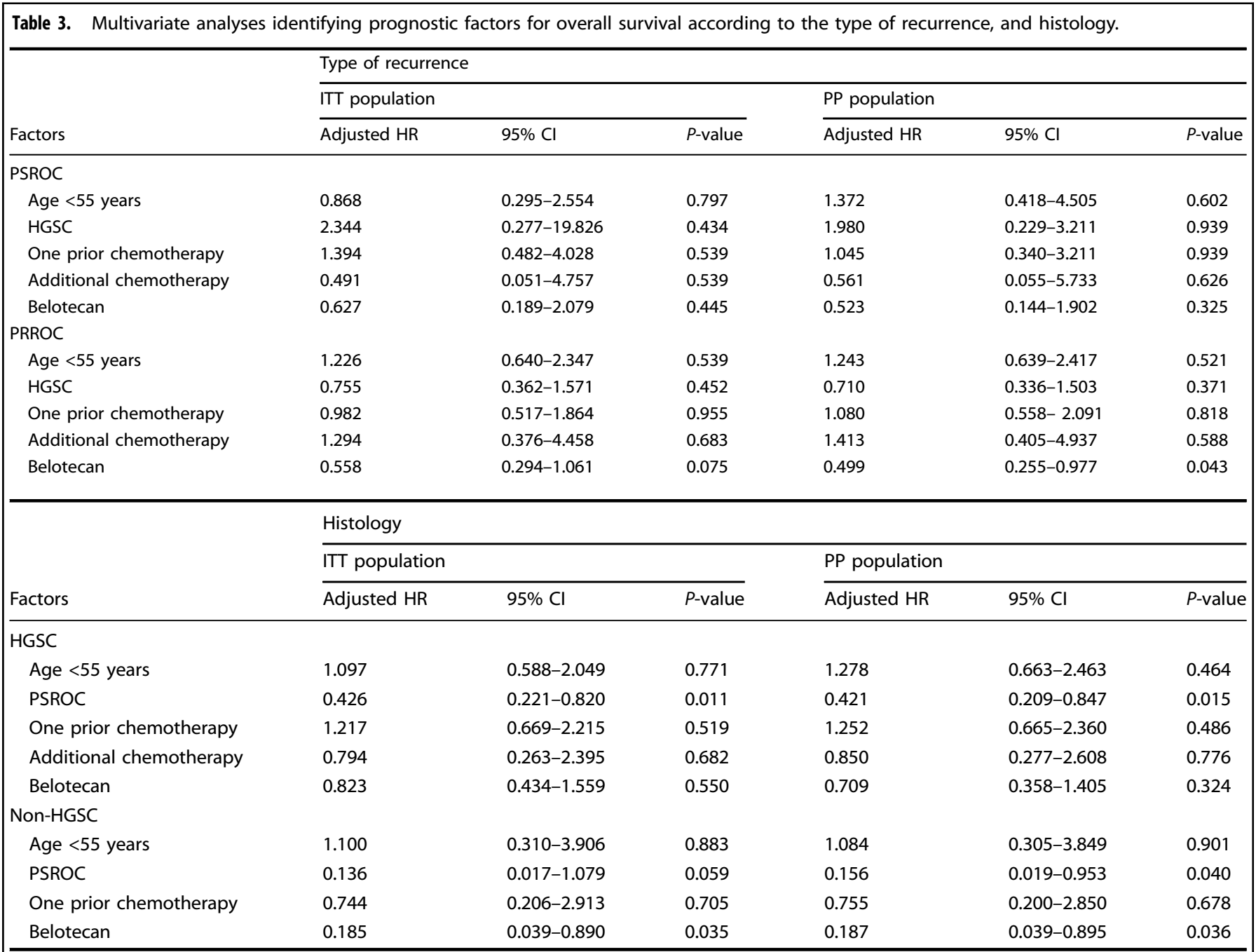

HGSC high-grade serous carcinoma, $H R$ hazard ratio, $C l$ confidence interval, $I T T$ intention-to-treat, $P P$ per-protocol, $P R R O C$ platinum-resistant recurrent ovarian cancer, PSROC platinum-sensitive recurrent ovarian cancer.

still important for combination therapy for treating PRROC in the era of targeted and immuno-oncologic therapy. ${ }^{23-26}$

In this study, belotecan showed similar ORR, PFS, and toxicity to topotecan. In particular, belotecan showed similar efficacy to topotecan as a topoisomerase I inhibitor for improving OS in patients with PSROC and HGSC, as indicated by there being no difference in OS between the two treatments in these subgroups. However, the ORR of belotecan was relatively low when compared with the result of our previous report where it was combined with cisplatin (38.5-41.7\% versus $78 \%$ ), suggesting that belotecan combined with cisplatin may be more effective than belotecan alone for PSROC. ${ }^{11}$

On the other hand, belotecan increased OS by 10.5-16 months compared with topotecan in patients with PRROC. These findings are meaningful when we considered that patients who received $>2$ prior chemotherapeutic regimens accounted for $10 \%$ of all patients, unlike the AURELIA trial, from which they were excluded, ${ }^{27}$ and that targeted or immune-oncologic drugs had not been administered in all patients after disease progression because these agents have been permitted since May 2005 in our country. ${ }^{28}$ Although there is no clear evidence indicating why belotecan may be associated with improved OS in PRROC, a preclinical study, where anti-tumour activity was compared between belotecan and topotecan without the addition of bevacizumab, has reported that anti-tumour activity of topotecan was not observed after $240 \mathrm{~min}$, whereas it was seen with belotecan for more than $250 \mathrm{~min}$ in various types of cancer cell lines, ${ }^{5}$ suggesting that the relatively long-lasting anti-tumour activity of belotecan can contribute to the improved OS when compared to topotecan.

Another interesting point is that belotecan was also associated with improved OS in patients with non-HGSC in the current study. Although we cannot explain this finding on the basis of evidence, we can suggest the hypothesis that belotecan may have more potential to increase T-cell-mediated cytotoxicity than topotecan because preclinical studies reported that topoisomerase I inhibitors may increase T-cell-mediated cytotoxicity depending on the upregulation of tumour protein 53-inducible nuclear protein 1 (TP53INP1) to positively regulate tumour cell apoptosis in melanoma cells. ${ }^{29,30}$ Especially, we found that patients with endometrioid or clear cell carcinoma who received belotecan were alive despite the death in $57.1 \%$ of the patients who received topotecan, suggesting improved OS by belotecan. Considering that TP53INP1 is upregulated in most of the patients with non-HGSC, the result supports the hypothesis that belotecan can further enhance T-cell-mediated cytotoxicity in comparison to topotecan, which should be proven in basic research. 


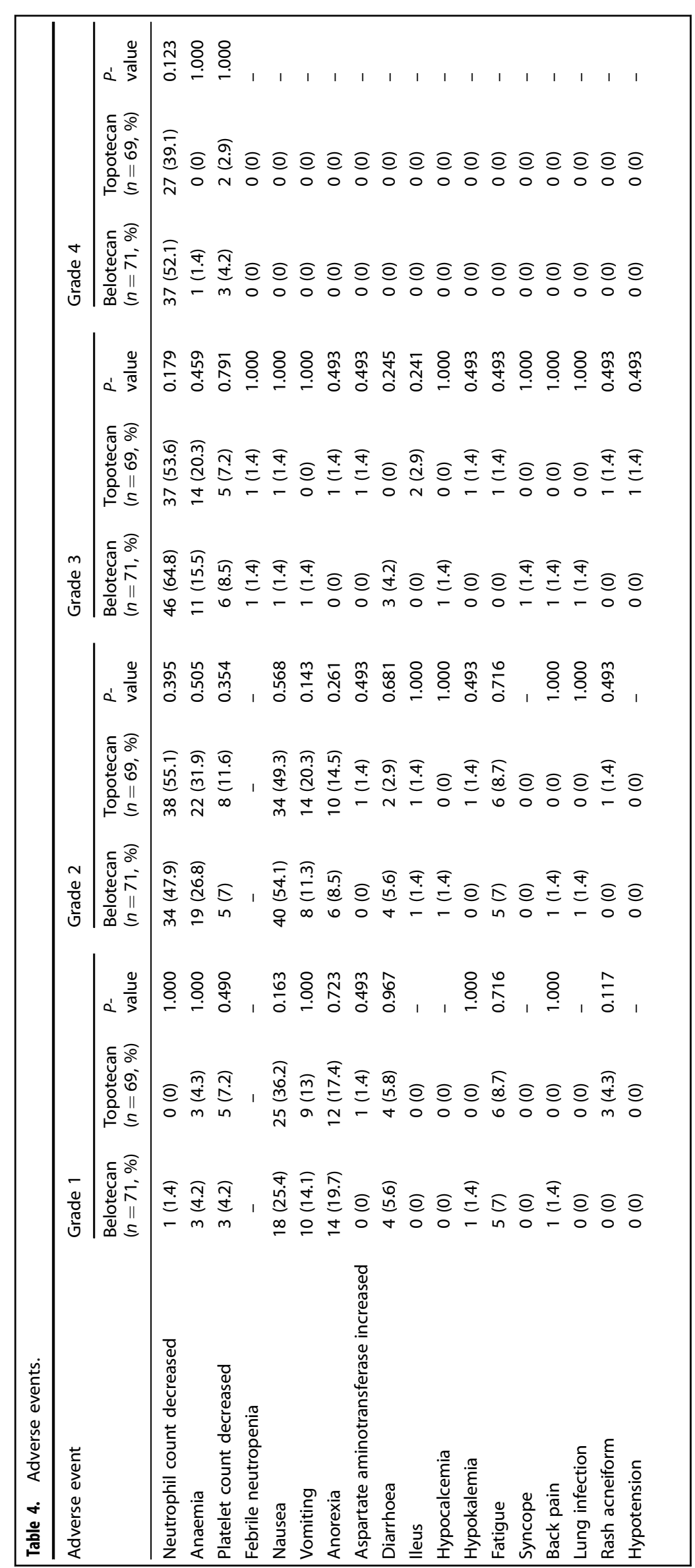


This study has several limitations that mean further trials are required to validate the findings. First, the result that belotecan was not inferior to topotecan in terms of overall response should be further investigated in well-designed studies because this study included both patients with PSROC and those with PRROC. Since patients with PSROC may show better ORR than those with PRROC in general, ORR between belotecan and topotecan should be compared in trials including each group. Second, we set ORR as the primary endpoint instead of OS and designed the study as a Phase $2 \mathrm{~b}$ trial. More clinical trials focused on OS is needed to prove that belotecan can improve OS. Third, patients with recurrent ovarian cancer were recruited heterogeneously regardless of the type of recurrence and histology. If belotecan may be more effective than topotecan, specifically in patients with PRROC or non-HGSC, new trials where only these patients are enrolled will be needed to prove this. Fourth, the potential of belotecan on better T-cell immunity or tumour response should be proven in basic research.

Despite these limitations, the study is meaningful because it suggests that belotecan maybe not inferior to topotecan in terms of overall response for recurrent ovarian cancer. Therefore, these results allow us to use belotecan for treating recurrent ovarian cancer in anticipation of treatment response comparable to topotecan.

\section{AUTHOR CONTRIBUTIONS}

H.S.K. and Y.S.S. conceived of the current study. H.S.K., S.-Y.P., C.-Y.P., Y.T.K., B.-J.K., Y.J.S., B.-G.K., Y.B.K., C.-H.C., J.H.K. and Y.S.S. designed the study and enrolled patients. H.S.K. and Y.S.S. analysed, interpreted data, and drafted the manuscript. All authors read and approved the manuscript for submission. Moreover, all authors approved the revised manuscript for publication.

\section{ADDITIONAL INFORMATION}

Ethics approval and consent to participate The Institutional Review Board (IRB) at Seoul National University Hospital (No. H-1011-035-339), National Cancer Center (No. NCCCTS-10-519), Gil Medical Center (No. GIRBA2426), Severance Hospital (No. 4-20100666), Korea Cancer Center Hospital (No. K-1102-001-001), Pusan National University Yangsan Hospital (No. 02-2013-014), Samsung Medical Center (No. SMC 2010-12-005), Seoul National University Bundang Hospital (No. B-1103-123-002), Dongsan Medical Center (No. 10-165) and Asan Medical Center (No. 2010-0813) approved the protocol version 1.0 for the current study. We enroled all patients in this study after we obtain their written consents.

Data availability A central manager had independent access to all data and materials. The central manager managed the database by sending queries about errors entered from each institution and having principal investigators or clinical research nurses make corrections to the entered data.

Competing interests S.-Y.P., C.-Y.P., Y.T.K., B.-J.K., Y.J.S., B.-G.K., Y.B.K., C.-H.C., J.H.K. and Y.S.S. received research funding from Chong Kun Dang Pharmaceutical, whereas HSK declares no conflict of interest.

Funding information This work was supported by Chong Kun Dang Pharmaceutical.

Supplementary information is available for this paper at https://doi.org/10.1038/ s41416-020-01098-8.

Note This work is published under the standard license to publish agreement. After 12 months the work will become freely available and the license terms will switch to a Creative Commons Attribution 4.0 International (CC BY 4.0).

Publisher's note Springer Nature remains neutral with regard to jurisdictional claims in published maps and institutional affiliations.

\section{REFERENCES}

1. Heintz, A. P., Odicino, F., Maisonneuve, P., Quinn, M. A., Benedet, J. L., Creasman, W. T. et al. Carcinoma of the ovary. FIGO 26th Annual Report on the Results of
Treatment in Gynecological Cancer. Int. J. Gynaecol. Obstet. 95(Suppl 1), S161-S192 (2006).

2. González Martín, A., Bratos, R., Márquez, R., Alonso, S. \& Chiva, L. Bevacizumab as front-line treatment for newly diagnosed epithelial cancer. Expert. Rev. Anticancer. Ther. 13, 123-129 (2013).

3. Markman, M., Markman, J., Webster, K., Zanotti, K., Kulp, B., Peterson, G. et al. Duration of response to second-line, platinum-based chemotherapy for ovarian cancer: implications for patient management and clinical trial design. J. Clin. Oncol. 22, 3120-3125 (2004).

4. Eisenhauer, E. A., Vermorken, J. B. \& van Glabbeke, M. Predictors of response to subsequent chemotherapy in platinum pretreated ovarian cancer: a multivariate analysis of 704 patients. Ann. Oncol. 8, 963-968 (1997).

5. Lee, J. H., Lee, J. M., Kim, J. K., Ahn, S. K., Lee, S. J. \& Kim, M. Y. et al. Antitumor activity of 7-[2-(N-isopropylamino)ethyl]-(20S)-camptothecin, CKD602, as a potent DNA topoisomerase I inhibitor. Arch. Pharm. Res. 21, 581-590 (1998).

6. Lee, J. H., Lee, J. M., Lim, K. H., Kim, J. K., Ahn, S. K., Bang, Y. J. et al. Preclinical and phase I clinical studies with $\mathrm{Ckd}-602$, a novel camptothecin derivative. Ann. N. Y. Acad. Sci. 922, 324-325 (2000).

7. Hsiang, Y. H., Lihou, M. G. \& Liu, L. F. Arrest of replication forks by drug-strabilized topoisomerase I-DNA clevable complexes as a mechanism of cell killing by camptothecin. Cancer Res. 49, 5077-5082 (1989).

8. Skipper, H. E., Schabel, F. M. Jr, Mellett, L. B., Montgomery, J. A., Wilkoff, L. J., Lloyd, H. H. et al. Implications of biochemical, cytokinetic, pharmacologic, and toxicologic relationships in the design of optimal therapeutic schedules. Cancer Chemother. Rep. 54, 431-450 (1970).

9. Sasaki, Y., Shinkai, T., Eguchi, K., Tamura, T., Ohe, Y., Ohmori, T. et al. Prediction of the antitumor activity of new platinum analogs based on their ex vivo pharmacodynamics as determined by bioassay. Cancer Chemother. Pharmacol. 27, 263-270 (1991).

10. Lee, H. P., Seo, S. S., Ryu, S. Y., Kim, J. H., Bang, Y. J., Park, S. Y. et al. Phase II evaluation of CKD-602, a camptothecin analog, administered on a 5-day schedule to patients with platinum-sensitive or -resistant ovarian cancer. Gynecol. Oncol. 109, 359-363 (2008).

11. Kim, H. S., Kang, S. B., Seo, S. S., Han, S. S., Kim, J. W., Park, N. H. et al. Phase I/lla study of combination chemotherapy with CKD-602 and cisplatin in patients with recurrent epithelial ovarian cancer. Ann. N. Y. Acad. Sci. 1171, 627-634 (2009).

12. Herzog, T. J., Sill, M. W., Walker, J. L., O'Malley, D., Shahin, M., DeGeest, K. et al. A phase II study of two topotecan regimens evaluated in recurrent platinumsensitive ovarian, fallopian tube or primary peritoneal cancer: a Gynecologic Oncology Group Study (GOG 146Q). Gynecol. Oncol. 120, 454-458 (2011).

13. McGuire, W. P., Blessing, J. A., Bookman, M. A., Lentz, S. S. \& Dunton, C. J. Topotecan has substantial antitumor activity as first-line salvage therapy in platinum-sensitive epithelial ovarian carcinoma: A Gynecologic Oncology Group Study. J. Clin. Oncol. 18, 1062-1067 (2000)

14. Sehouli, J., Stengel, D., Harter, P., Kurzeder, C., Belau, A., Bogenrieder, T. et al. Topotecan weekly versus conventional 5 -day schedule in patients with platinumresistant ovarian cancer: a randomized multicenter phase II trial of the NorthEastern German Society of Gynecological Oncology Ovarian Cancer Study Group. J. Clin. Oncol. 29, 242-248 (2011).

15. Swisher, E. M., Mutch, D. G., Rader, J. S., Elbendary, A. \& Herzog, T. J. Topotecan in platinum- and paclitaxel-resistant ovarian cancer. Gynecol. Oncol. 66, 480-486 (1997).

16. Eisenhauer, E. A., Therasse, P., Bogaerts, J., Schwartz, L. H., Sargent, D., Ford, R. et al. New response evaluation criteria in solid tumours: revised RECIST guideline (version 1.1). Eur. J. Cancer 45, 228-247 (2009).

17. Rustin, G. J., Vergote, I., Eisenhauer, E., Pujade-Lauraine, E., Quinn, M., Thigpen, T. et al. Definitions for response and progression in ovarian cancer clinical trials incorporating RECIST 1.1 and CA 125 agreed by the Gynecological Cancer Intergroup (GCIG). Int. J. Gynecol. Cancer 21, 419-423 (2011).

18. Common Terminology Criteria for Adverse Events (CTCAE) v4.0. https://ctep. cancer.gov/protocolDevelopment/electronic_applications/ctc.htm\#ctc_40. Accessed 1 Feb 2020.

19. Bookman, M. A., Malmström, H., Bolis, G., Gordon, A., Lissoni, A., Krebs, J. B. et al. Topotecan for the treatment of advanced epithelial ovarian cancer: an open-label phase II study in patients treated after prior chemotherapy that contained cisplatin or carboplatin and paclitaxel. J. Clin. Oncol. 16, 3345-3352 (1998).

20. Kudelka, A. P., Tresukosol, D., Edwards, C. L., Freedman, R. S., Levenback, C., Chantarawiroj, P. et al. Phase II study of intravenous topotecan as a 5-day infusion for refractory epithelial ovarian carcinoma. J. Clin. Oncol. 14, 1552-1557 (1996).

21. Pujade-Lauraine, E., Ledermann, J. A., Selle, F., Gebski, V., Penson, R. T., Oza, A. M. et al. Olaparib tablets as maintenance therapy in patients with platinum-sensitive, relapsed ovarian cancer and a BRCA1/2 mutation (SOLO2/ENGOT-Ov21): a double-blind, randomised, placebo-controlled, phase 3 trial. Lancet Oncol. 18, 1274-1284 (2017). 
22. Coleman, R. L., Brady, M. F., Herzog, T. J., Sabbatini, P., Armstrong, D. K., Walker, J. L. et al. Bevacizumab and paclitaxel-carboplatin chemotherapy and secondary cytoreduction in recurrent, platinum-sensitive ovarian cancer (NRG Oncology/ Gynecologic Oncology Group study GOG-0213): a multicentre, open-label, randomised, phase 3 trial. Lancet Oncol. 18, 779-791 (2017).

23. Chekerov, R., Hilpert, F., Mahner, S., El-Balat, A., Harter, P., De Gregorio, N. et al. Sorafenib plus topotecan versus placebo plus topotecan for platinum-resistant ovarian cancer (TRIAS): a multicentre, randomised, double-blind, placebo-controlled, phase 2 trial. Lancet Oncol. 19, 1247-1258 (2018).

24. Musa, F., Pothuri, B., Blank, S. V., Ling, H. T., Speyer, J. L., Curtin, J. et al. Phase II study of irinotecan in combination with bevacizumab in recurrent ovarian cancer. Gynecol. Oncol. 144, 279-284 (2017).

25. Kurzeder, C., Bover, I., Marmé, F., Rau, J., Pautier, P., Colombo, N. et al. Doubleblind, placebo-controlled, randomized phase III trial evaluating pertuzumab combined with chemotherapy for low tumor human epidermal growth factor receptor 3 mRNA-expressing Platinum-Resistant Ovarian Cancer (PENELOPE). J. Clin. Oncol. 34, 2516-2525 (2016).
26. Pujade-Lauraine, E., Hilpert, F., Weber, B., Reuss, A., Poveda, A., Kristensen, G. et al. Bevacizumab combined with chemotherapy for platinum-resistant recurrent ovarian cancer: The AURELIA open-label randomized phase III trial. J. Clin. Oncol. 32, 1302-1308 (2014).

27. Poveda, A. M., Selle, F., Hilpert, F., Reuss, A., Savarese, A., Vergote, I. et al. Bevacizumab combined with weekly paclitaxel, pegylated liposomal doxorubicin, or topotecan in platinum-resistant recurrent ovarian cancer: Analysis by Chemotherapy Cohort of the Randomized Phase III AURELIA Trial. J. Clin. Oncol. 33, 3836-3838 (2015)

28. Park, S. J., Kim, J., Kim, S. N., Lee, E. J., Oh, S., Seol, A. et al. Practice patterns of surgery for advanced ovarian cancer: analysis from international surveys. Jpn. J. Clin. Oncol. 49, 137-145 (2019).

29. Okamura, S., Arakawa, H., Tanaka, T., Nakanishi, H., Ng, C. C., Taya, Y. et al. p53DINP1, a p53-inducible gene, regulates p53-dependent apoptosis. Mol. Cell. 8, 85-94 (2001).

30. McKenzie, J. A., Mbofung, R. M., Malu, S., Zhang, M., Ashkin, E., Devi, S. et al. The effect of topoisomerase I inhibitors on the efficacy of T-cell-based cancer immunotherapy. J. Nat/ Cancer Inst. 110, 777-786 (2018). 